\title{
Ethnic Disparities in Coronavirus Disease 2019 after the Implementation of Universal Screening in Hartford, Connecticut
}

\author{
Veronica Maria Pimentel, MD, MS 1,2,3(0) \\ Reinaldo Figueroa, $\mathrm{MD}^{1,2 \odot}$ \\ ${ }^{1}$ Department of Obstetrics and Gynecology, St. Francis Hospital and \\ Medical Center/Trinity Health of New England, Hartford, Connecticut \\ 2 Department of Obstetrics and Gynecology, Quinnipiac University, \\ Frank H. Netter School of Medicine, North Haven, Connecticut \\ ${ }^{3}$ Department of Obstetrics and Gynecology, University of \\ Connecticut School of Medicine, Farmington, Connecticut \\ ${ }^{4}$ Division of Obstetrics and Gynecology, University of New England, \\ College of Osteopathic Medicine, Biddeford, Maine
}

\author{
Frank lan Jackson, $\mathrm{DO}^{1,4}$ Anthony Dino Ferrante, $\mathrm{DO}^{1}$
}

\begin{abstract}
Address for correspondence Veronica Maria Pimentel, MD, MS, Department of Obstetrics and Gynecology, Saint Francis Hospital and Medical Center, 114 Woodland Street, Hartford, CT 06105 (e-mail: veronicamaria.pimentel@trinityhealthofne.org).
\end{abstract}

AJP Rep 2021;11:e147-e153.

\begin{abstract}
Keywords

- coronavirus

- COVID-19

- SARS-CoV-2

- pregnancy

- ethnic disparities

- obstetric outcomes

- neonates

- labor and delivery

Objective The aim of this article was to estimate the prevalence of coronavirus disease 2019 (COVID-19) in Connecticut, examine racial/ethnic disparities, and assess pregnancy outcomes in pregnant women following the implementation of universal screening for the virus.

Materials and methods This is a retrospective cohort study of all obstetric patients admitted to our labor and delivery unit during the first 4 weeks of implementation of universal screening of COVID-19. Viral studies were performed in all neonates born to mothers with severe acute respiratory syndrome coronavirus 2 . We calculated the prevalence of COVID-19, compared the baseline characteristics and pregnancy outcomes between those who tested positive and negative for the virus, and determined the factors associated with COVID-19.

Results A total of 10 (4.6\%) of 220 women screened positive for the virus. All were asymptomatic. Week 1 had the highest prevalence of infection, nearing $8 \%$. No neonates were infected. Hispanics were more likely to test positive (odds ratio: 10.23; confidence interval: [2.71-49.1], $p=0.001)$. Obstetric and neonatal outcomes were similar between the groups $(p>0.05)$.

Conclusion Although the rate of asymptomatic COVID-19 was low, ethnic disparities were present with Hispanics being more likely to have the infection.
\end{abstract}

\section{Key Points}

- $4.6 \%$ of pregnant women in labor and delivery tested positive for COVID-19 while being asymptomatic.

- Hispanic women were more likely to test positive for severe acute respiratory syndrome coronavirus 2.

- Pregnancy outcomes were similar between COVID-19 positive and negative women.

- No vertical transmission was detected.

received

August 17, 2020 accepted after revision October 8, 2021
DOI https://doi.org/ 10.1055/s-0041-1740562. ISSN 2157-6998.

\footnotetext{
(C) 2021. The Author(s).

This is an open access article published by Thieme under the terms of the Creative Commons Attribution-NonDerivative-NonCommercial-License, permitting copying and reproduction so long as the original work is given appropriate credit. Contents may not be used for commercial purposes, or adapted, remixed, transformed or built upon. (https://creativecommons.org/ licenses/by-nc-nd/4.0/)

Thieme Medical Publishers, Inc., 333 Seventh Avenue, 18th Floor, New York, NY 10001, USA
} 
Severe acute respiratory syndrome coronavirus 2 (SARSCoV-2) has spread through communities worldwide, including Hartford, Connecticut (CT), causing a novel coronavirus disease known as coronavirus disease 2019 (COVID-19). There is still limited data about the impact of this virus in pregnancy. However, pregnant women are known to be at greater risk of severe morbidity and mortality from other respiratory infections such as influenza ${ }^{1}$ and SARS-CoV- $1 .{ }^{2} \mathrm{~A}$ systematic review reported on 19 studies that included 79 pregnant women with confirmed coronavirus spectrum illness, 41 women were affected by COVID-19. ${ }^{2}$ In this population, overt pneumonia was diagnosed in $91.8 \%$ of the women, and the most common symptoms were fever (82.6\%), cough (57.1\%), and dyspnea (27\%). For COVID-19 patients specifically, the rate of preterm birth $(<37$ weeks) was $41.1 \%$, preterm premature rupture of membranes was $18.8 \%$, perinatal death was $7 \%, 91 \%$ of pregnancies were delivered by cesarean, and $10 \%$ of women were admitted to the intensive care unit (ICU).

Non-Hispanic Blacks and Hispanic/Latinx have been disproportionately impacted by COVID-19 in many areas of the United States. A serologic study in New York State demonstrated non-Hispanic Blacks and Hispanics were much more likely to test positive for COVID-19 antibodies than people of other racial and ethnic backgrounds. ${ }^{3}$ Additionally, Black patients with COVID-19 were noted to have a higher prevalence of comorbid conditions such as kidney failure, diabetes mellitus, hypertension, and obesity, all of which are associated with a higher likelihood of a need for admission. ${ }^{4}$ Further studies supported the increased risk of hospitalization for non-Hispanic Black patients and also demonstrated an increased likelihood of death. ${ }^{5}$

Our understanding of the virus is incomplete and constantly evolving; therefore, we need more data on the prevalence of this condition. To protect our patients and caregivers, we need to improve our understanding of the impact of this virus on the health of the mother, in the antepartum and postpartum periods, and of the fetus and newborn. Studies of symptomatic patients with COVID-19 likely underestimate the prevalence of the virus in the pregnant population as two studies from New York reported the prevalence of SARS-CoV-2 symptoms as $1.9^{6}$ and $6.8 \%{ }^{7}$ in pregnant patients while the prevalence of asymptomatic infection was similar at $13.5^{6}$ and $13 \%{ }^{7}$ respectively. More recently, in Southern CT (Yale New Haven Health system), of the 30 of 770 women (3.9\%) who tested positive for the virus, only $8(1.0 \%)$ were symptomatic. ${ }^{8}$ In CT, Hispanics/Latinx and Blacks have accounted for a disproportionate number of COVID-19 patients. $^{9}$ Data from large metropolitan areas, such as Boston, Massachusetts and New York City (NYC), New York, have demonstrated racial and ethnic disparities in COVID-19. ${ }^{10,11}$ However, we found no published studies about these disparities in smaller cities. We do not know the prevalence of COVID-19 in the pregnant population of Northern CT and if racial/ethnic disparities exist. Thus, our study aimed to estimate the prevalence of COVID-19 in pregnant women in Hartford, CT, following the implemen- tation of universal screening for the virus and then examine if racial and/or ethnic disparities existed.

On April 24, 2020, Saint Francis Hospital and Medical Center (SFHMC) of Trinity Health Of New England (THONE) implemented universal screening of COVID-19 with a nasopharyngeal swab obtained from all women admitted to the labor and delivery (L\&D) unit. Our primary objective was to evaluate the prevalence of COVID-19 in symptomatic and asymptomatic pregnant women for the first 28 days since the implementation of universal screening of COVID-19 and determine if there were any racial and/or ethnic disparities in COVID-19 in pregnancy. A secondary objective was to assess the pregnancy outcomes of women who screened positive for COVID-19 and compare them to those who screened negative.

\section{Materials and Methods}

This is a retrospective cohort study of all obstetric patients admitted to the SFHMC L\&D unit from April 24, 2020 to May 21, 2020, corresponding to the first 4 weeks of implementation of universal screening of COVID-19. Testing was performed in all patients admitted, regardless of indication for admission or presence of symptoms. Institutional review board approval was obtained for this study.

All patients were asked about symptoms associated with COVID-19 (fever, cough, shortness of breath, myalgia, fatigue, and loss of taste and smell) at the time of presentation to L\&D by a registered nurse, and asked if they had these symptoms within the last 6 weeks, been exposed to someone with suspected or confirmed COVID-19, traveled to areas deemed high risk at the time (New York, Washington State, Oregon, California, or out of the United States). Sampling was performed by trained nurses in appropriate personal protective equipment using a nasopharyngeal swab, which was collected in the multi-collect specimen collection kit for viruses and sent to our hospital laboratory for analysis of SARS-CoV-2. SARS-CoV-2 viral studies were performed in all neonates born to mothers with a positive COVID-19 test.

Patient baseline demographics, including race and ethnicity, medical history and clinical course, were obtained from the electronic medical record. The overall prevalence of COVID-19 was calculated using the number of patients who tested positive divided by the number of all patients who underwent testing during the 4-week period. The prevalence of COVID-19 was also calculated per week. Pregnancy outcomes of those who tested positive (positive group) were compared with those of patients who tested negative (negative group). $X^{2}$ analyses were done for categorical variables, Student $t$-test for continuous variables, and Wilcoxon rank sum test for noncontinuous variables. Binomial regression was used to determine factors associated with COVID-19; variables that were not significantly associated with COVID-19 were not included in a final model assessing for the association between COVID-19 and ethnicity. All analyses were performed using the Rersion 3.6.0. ${ }^{12} \mathrm{~A}$ $p<0.05$ was considered significant. 


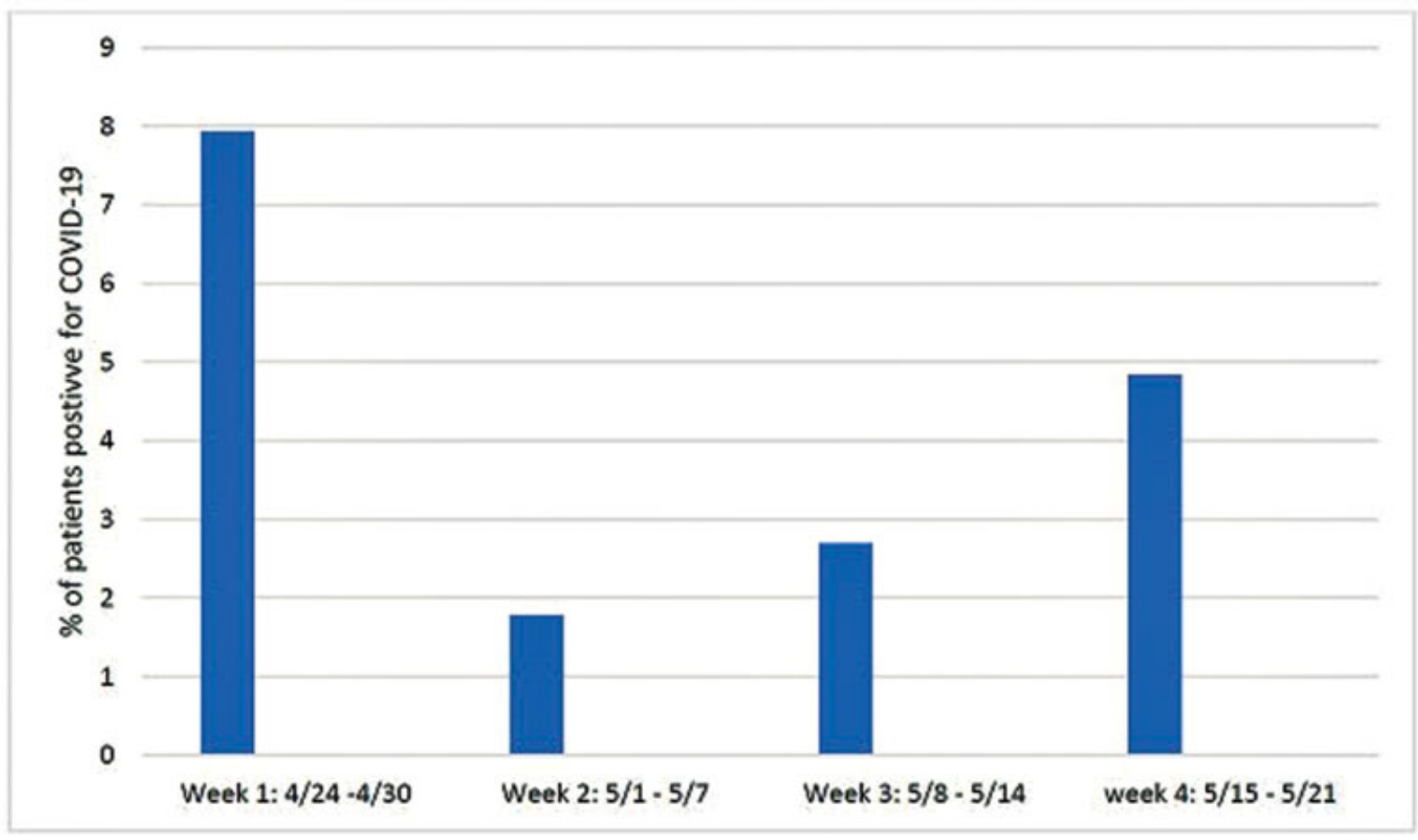

Fig. 1 Prevalence of coronavirus disease 2019 (COVID-19) by weeks of testing in labor and delivery.

\section{Results}

Two hundred twenty women were screened for COVID-19 from April 24, 2020 to May 21, 2020. Ten patients tested positive corresponding to a rate of $4.6 \%$. - Fig. 1 shows that the positive rate of COVID-19 varied by week, with week 1 having the highest rate of positive results nearing $8 \%$. None of the neonates tested positive for this virus 24 hours after delivery.

All patients were asymptomatic at the time testing was performed in L\&D. Patients who tested positive for COVID-19 had an oxygen saturation that ranged from 97 to $100 \%$ on admission. One patient had presented to the emergency department 1 month earlier with symptoms of cough, which had resolved prior to her L\&D admission while her nasopharyngeal polymerase chain reaction remained positive for SARS-CoV-2. - Table 1 shows that the positive group was more likely to identify their race as "other" $(p=0.002)$ and ethnicity as Hispanic $(p<0.001)$. There was no difference between the two groups in terms of body mass index, parity, gestational age, comorbidities (chronic hypertension, preeclampsia, asthma, gestational hypertension, pregestational diabetes), and multiple gestation ( $p>0.05)$.

- Table 2 shows that there was no association between testing positive for SARS-CoV-2 virus and maternal comorbidities $(p>0.05)$. Racial identification as "other" and being Hispanic were associated with an increased risk of testing positive for SARS-CoV-2 virus (odds ratio [OR]: 6.38; confidence interval [CI]: [1.39-44.9], $p=0.02$ and OR: 10.23; $\mathrm{CI}$ : [2.71-49.1], $p=0.001$, respectively). However, 38/46 (82.6\%) of Hispanics racially identified as "other" and 38/45 (84.4\%) of patients identifying as "other" were Hispanics. Older age was associated with a decreased risk of testing positive for the virus (OR: 0.83; $\mathrm{Cl}$ : [0.73-0.95], $p=0.006$ ).

- Table 3 shows that after controlling for age, Hispanic ethnicity remained significantly associated with an increased risk of testing positive for the virus (OR: 6.74; CI: [1.66-34.11], $p=0.01$ ).

- Table 4 shows that the rate of pregnancy complications such as preterm birth, postpartum hemorrhage, preeclampsia, cesarean delivery, and primary cesarean delivery in the nulliparous term singleton vertex patient was similar between the two groups $(p>0.05)$. Additionally, the rate of fetal/neonatal outcomes such as fetal demise, neonatal death, and admission to the neonatal ICU was similar between the two groups ( $p>0.05)$, except for one (10\%) previable birth in the positive group compared with none in the negative group $(p<0.001)$. There were no maternal admissions to the ICU, and no maternal or neonatal deaths in either group.

\section{Conclusion}

This is the first study to assess the prevalence of COVID-19 in Northern CT. It showed that the prevalence of positive SARS$\mathrm{CoV}-2$ tests in asymptomatic pregnant women presenting to the $L \& D$ unit was $4.6 \%$. This is also the first study to report on the ethnic disparities in COVID-19 in pregnancy in CT and in a smaller urban center. It highlighted the disproportionate representation of women of Hispanic/Latinx origin in the positive group. In general, maternal and neonatal outcomes were similar between mothers who tested positive and those 
Table 1 Baseline characteristics of pregnant women tested for COVID-19

\begin{tabular}{|l|l|l|l|}
\hline \multirow{2}{*}{} & COVID-19 status & $p$-Value \\
\cline { 2 - 4 } & Positive $\boldsymbol{n}=\mathbf{1 0}$ & Negative $\boldsymbol{n}=\mathbf{2 1 0}$ & \\
\hline Age, $y$ & $24.5(19-37)$ & $30(17-41)$ & 0.053 \\
\hline Body mass index, $\mathrm{kg} / \mathrm{m}^{2}$ & $31.7(23.8-64)$ & $32.6(21.1-70)$ & 0.859 \\
\hline Race/ethnicity, $n$ (\%) & & & \\
\hline Asian & $0(0)$ & $37(17.6)$ & Unable to calculate \\
\hline Black/African American & $2(20)$ & $51(24.3)$ & 0.757 \\
\hline White & $2(20)$ & $83(39.5)$ & 0.215 \\
\hline Other & $6(60)$ & $39(18.6)$ & 0.002 \\
\hline Ethnicity & & & \\
\hline Hispanic & $7(70)$ & $39(18.6)$ & $<0.001$ \\
\hline Nulliparous, $n$ (\%) & $4(40)$ & $75(35.7)$ & 0.780 \\
\hline Gestational week at testing & $39(18-40)$ & $38(27-41)$ & 0.722 \\
\hline Chronic hypertension, $n$ (\%) & $0(0)$ & $17(8.1)$ & 0.349 \\
\hline Hypertensive disorders of pregnancy, $n(\%)$ & $0(0)$ & $18(8.6)$ & 0.334 \\
\hline Asthma, $n$ (\%) & $1(10)$ & $39(18.6)$ & 0.492 \\
\hline Pregestational diabetes, $n$ (\%) & $0(0)$ & $5(2.5)$ & 0.622 \\
\hline Twins, $n$ (\%) & $0(0)$ & $13(6.2)$ & 0.417 \\
\hline
\end{tabular}

Abbreviation: COVID-19, coronavirus disease 2019.

Note: Categorical variables, chi-squared test. Continuous variables, independent Student's t-test.

who tested negative, except one mother in the positive group had a second trimester previable birth. All the neonates from positive mothers tested negative for SARS-CoV-2.

Studies of symptomatic patients with COVID-19 likely underestimate the prevalence of the virus in the pregnant population. This study was started after the initial peak of the pandemic when Hartford County had a rate of 150 COVID-19 cases per week per 100,000 population. In Southern CT, three

Table 2 Odds ratios of demographic and comorbid conditions with COVID-19

\begin{tabular}{|c|c|c|}
\hline & OR $[95 \% \mathrm{Cl}]$ & $p$-Value \\
\hline Hypertensive disease $^{a}$ & $0[0 \rightarrow 99]$ & 0.99 \\
\hline Diabetic disease $^{a}$ & $0[0 \rightarrow 99]$ & 0.95 \\
\hline Asthma & $0.49[0.03-2.70]$ & 0.50 \\
\hline Obesity & $0.45[0.09-2.17]$ & 0.31 \\
\hline Advanced maternal age & $0.39[0.02-2.10]$ & 0.81 \\
\hline Age & $0.83[0.73-0.95]$ & 0.006 \\
\hline Black/African American & $1.62[0.19-13.9]$ & 0.63 \\
\hline Other & 6.38 [1.39-44.9] & 0.02 \\
\hline Asian $^{a}$ & $0[0 \rightarrow 99]$ & 0.99 \\
\hline Hispanic & $10.23[2.71-49.1]$ & 0.001 \\
\hline
\end{tabular}

Abbreviation: $\mathrm{Cl}$, confidence interval; COVID-19, coronavirus disease 2019; OR, odds ratio.

${ }^{a}$ No woman with hypertension, diabetes, or woman of Asian descent were found to have COVID-19 explaining the OR range from 0 to greater than 99. hospitals affiliated with the Yale New Haven Health system reported a lower prevalence of $2.9 \%$ (22/756) for positive results among asymptomatic patients admitted for childbirth but did not comment on ethnic or racial disparities. ${ }^{8}$ In their study, conducted from April 2, 2020 to April 29, 2020 at the peak of the pandemic in CT, 73.3\% (22/30) were asymptomatic. New Haven County had a rate of 205 COVID-19 cases per week per 100,000 population. As of August 10, 2020, the total of laboratory-confirmed and probable COVID-19 cases reported among CT residents was 50,684, of which 12,826 came from Hartford County and 13,240 came from New Haven County. However, Hartford County had 1,415 and New Haven County had 1,106 COVID-19 associated deaths. ${ }^{9}$

NYC, the epicenter of the pandemic in the United States and worldwide in the spring of 2020, had a higher prevalence of positive tests in asymptomatic pregnant patients presenting to the L\&D unit. In two NYC hospitals associated with Columbia University, $15.4 \%$ of 215 women tested positive for SARS-CoV-2 when admitted for delivery; $13.5 \%$ were asymptomatic while $1.9 \%$ were symptomatic. ${ }^{6}$ In other words,

Table 3 Association of COVID-19 with Hispanic ethnicity after controlling for age

\begin{tabular}{|l|l|l|}
\hline & OR $(95 \% \mathrm{CI})$ & $p$-Value \\
\hline Hispanic & $6.74[1.66-34.11]$ & 0.01 \\
\hline Age & $0.88[0.75-1.02]$ & 0.098 \\
\hline
\end{tabular}

Abbreviation: $\mathrm{Cl}$, confidence interval; COVID-19, coronavirus disease 2019; OR, odds ratio. 
Table 4 Association of COVID-19 with maternal and fetal/neonatal outcomes

\begin{tabular}{|l|l|l|l|}
\hline \multirow{2}{*}{} & \multicolumn{2}{|l|}{ COVID-19 status } & $p$-Value \\
\cline { 2 - 4 } & Positive, $\boldsymbol{n}=\mathbf{1 0}$ & Negative, $\boldsymbol{n}=\mathbf{2 1 0}$ & \\
\hline Maternal outcomes & & & \\
\hline Preterm delivery, $n$ (\%) & $1(10)$ & $35(16.7)$ & 0.578 \\
\hline Postpartum hemorrhage, $n(\%)$ & $0(0)$ & $17(8.1)$ & 0.349 \\
\hline Intrauterine infection, $n(\%)$ & $0(0)$ & $8(3.8)$ & 0.530 \\
\hline Preeclampsia with severe features, $n(\%)$ & $0(0)$ & $27(12.9)$ & 0.226 \\
\hline Maternal ICU admission, $n(\%)$ & $0(0)$ & $0(0)$ & Unable to calculate \\
\hline NTSV cesarean delivery $n(\%)$ & $20(32.3)$ & 0.599 \\
\hline Overall cesarean delivery, $n(\%)$ & $1(50)$ & $114(54.3)$ & 0.791 \\
\hline Fetal/neonatal outcomes & $5(50)$ & & \\
\hline NICU admission, $n$ (\%) & & $34(16.2)$ & 0.601 \\
\hline Neonatal death, $n$ (\%) & $1(10)$ & $0(0)$ & Unable to calculate \\
\hline Fetal demise, $n$ (\%) & $0(0)$ & $1(0.4)$ & 0.827 \\
\hline Previable birth, $n$ (\%) & $0(0)$ & $0(0)$ & $<0.001$ \\
\hline COVID-19 in neonate & $1(10)$ & Not tested & \\
\hline
\end{tabular}

Abbreviations: COVID-19, coronavirus disease 2019; ICU, intensive care unit; NICU, neonatal intensive care unit; NTSV, nulliparous term singleton vertex.

${ }^{*}$ Of NTSV patients, 2 tested positive for COVID-19 and 62 tested negative.

87.9\% (29/33) of the patients who were positive for SARSCoV-2 at admission had no symptoms. The study was conducted between March 22 and April 4, 2020 and NYC already had 10,764 positive cases and 99 deaths by March 22, 2020. ${ }^{13}$ At NYU Winthrop Hospital in Nassau County, Long Island, $19.9 \%$ of 161 women tested positive for COVID-19 when admitted for delivery and $66 \%(21 / 32)$ of the positive patients lacked any reported symptoms of COVID-19.7 Thirteen percent of the population tested positive and were asymptomatic. The study was done from March 30 to April 12, 2020 and Nassau County had already 4,657 positive cases and 27 deaths by March 27, 2020. ${ }^{14}$

Our study had a disproportionate representation of Hispanic/Latinx patients in the COVID-19 positive group. In $C T$, people who identify as Hispanic/Latinx and nonHispanic Blacks, as of August 10, 2020, had a much higher age-adjusted prevalence of COVID-19 with 1,839 and 1,739 cases per 100,000 population, respectively, compared with non-Hispanic whites with 571 cases per 100,000 population. ${ }^{9}$ This finding in conjunction with our study raises the concern that COVID-19 is disproportionally affecting people of Hispanic/Latinx origin in Northern, CT, and suggests health disparities that need to be addressed. Additionally, our study supports that ethnic disparities in the rate of diagnosis in the general population may reflect the rate of diagnosis within pregnant women. In term of racial disparities, the only significant difference we found was an increased risk of COVID-19 in pregnant women classified racially as "other," but ethnically considered themselves Hispanic/Latinx. Surprisingly, our study did not find a significant difference in COVID-19 between African-American/
Black women and White women despite the larger prevalence of COVID-19 in the African-American/Black community.

More studies are needed to assess the reason behind the ethnic disparities in COVID-19 pregnancy. Reports from Boston and NYC on ethnic disparities associated with COVID-19 in pregnancy have shown a disproportionate representation of Hispanic women. ${ }^{10,11}$ In Boston, Goldfarb et al prospectively studied 192 women who were pregnant or had recently delivered with symptoms of COVID-19 infection from March 6 to May 4, 2020. Of the 136 women that were tested, $72 \%$ (39/54) of the Hispanic women and 27\% (22/82) non-Hispanic women were positive for COVID-19. The majority of the Hispanic women had public insurance (79\%) and their primary language was not English (74\%), while the majority of the non-Hispanic women had private insurance (73\%) and English was the primary language in $91 \%{ }^{10}$ In NYC, Emeruwa et al retrospectively studied 100 women who tested positive for SARS-Cov-2 infection among 673 women who delivered from March 13 to April 23, 2020. While $9.4 \%(13 / 138)$ of the non-Hispanic White women tested positive, $18.1 \%$ (73/403) of the Hispanic women tested positive for SARS-CoV-2 infection. ${ }^{11}$ In addition, $82 \%$ of Hispanic women had public insurance, and $49 \%$ came from the Bronx and 38\% from Manhattan (boroughs that were significantly affected by COVID-19 infection). In a population-based study on the general population in Massachusetts, Figueroa et al found that household size, employment as a food service worker, and number of noncitizens were associated with higher rates in the Hispanic/Latinx community. ${ }^{15}$ We did not assess the socioeconomic status of our 
study population. However, it is likely that the CT Hispanic/Latinx families in our study faced similar challenges to those in the neighboring states of Massachusetts and New York.

While health disparities in the United States have been well established, these findings have yet to be demonstrated in pregnant women in other regions of the United States, likely due to the disease being more prevalent in the Northeast at the time of this study. With states in the southern US being significantly more impacted in the summer of 2020 , other hospitals should be able to demonstrate whether women of Hispanic/Latinx origin are more likely to be impacted by COVID-19 or if this was an effect limited to the Northeast. Additionally, there remains a paucity of data on racial and ethnic contributors to healthcare outcomes in pregnancy in other countries. Data from France show that people born in Africa are twice as likely to die from COVID-19 than people born in Europe, ${ }^{16}$ highlighting that this virus disproportionately impacts marginalized communities. These disparities are not inherent to a race or ethnicity. Rather, they are a result of systemic racism that has caused social, political, and economic marginalization of different populations in different states and countries. We need a better understanding of the significance of differences in risk of infection from work exposure, household transmission, and community contact. ${ }^{17}$ In addition, we need to study the differences in illness severity, not just the presence of comorbidities, which may have arisen from the lack of access to care, lack of insurance, or lack of money. ${ }^{17}$ Moreover, there is a need to study the quality of care given when patients have access to care. It is time for the scientific and medical community to evaluate more in depth the effect of systemic racism on outcomes and implement solutions that will end health disparities.

In general, our asymptomatic positive patients had similar obstetric outcomes as the negative patients. Therefore, mode of delivery should be based on obstetric indications. Additional studies from other parts of the country are needed to corroborate our findings. Importantly, the long-term impact of this virus on mothers and their offspring remains unknown. However, we anticipate that families of Hispanic/Latinx origin would be more likely to be impacted and this can lead to significant future health disparities.

In the NYC study by Sutton et al, ${ }^{6} 10.3 \%$ (3/29) of the asymptomatic positive patients developed symptoms in the postpartum period. In our study, none of the asymptomatic patients who tested positive developed symptoms while hospitalized. Neither the study by Vintzileos et $\mathrm{al}^{7}$ nor the New Haven study by Campbell et $\mathrm{al}^{8}$ reported this information. It is plausible that the three patients who developed symptoms likely presented to the hospital with a subclinical active infection, while our patients did not develop symptoms as they were in the convalescent period of the infection judging by the period when the studies were performed.

One of our patients that tested positive for COVID-19 had a second trimester previable birth. Although the placenta was not tested for the presence of SARS-CoV-2, it did not show any typical pathological signs of infection. Not much information is available regarding infections that occur in the second trimester. A report of a pregnancy loss at 19 weeks of gestation on a patient with symptomatic COVID-19 revealed findings of the virus in the placental cotyledon and submembrane. The umbilical cord blood, amniotic fluid, and fetus tested negative. ${ }^{18}$

Strengths of the study include the testing of all patients that came to the L\&D unit during the study period. We were able to compare the obstetric outcomes of patients who tested positive with patients who tested negative and show that the obstetric outcomes were similar. Limitations of the study include its retrospective nature and reliance on accurate documentation. The sample size was small and we had only 10 patients that tested positive. Our study was conducted as the rate of COVID-19 was starting to decline in Hartford County, which likely affected our positive rate.

In conclusion, 10 out of 220 pregnant women presenting to L\&D tested positive for SARS-CoV-2 virus while being asymptomatic for COVID-19. However, their obstetric and neonatal outcomes were similar to the pregnant women that tested negative. There did not appear to be vertical transmission in asymptomatic SARS-CoV-2 positive patients during the third trimester in our institution. Finally, Hispanic women were more likely to test positive for SARS-CoV-2, suggesting a need to address health disparities in COVID-19.

Conflict of Interest

None declared.

\section{References}

1 Immunization Infectious Diseases ACOG and Public Health Work Group Swamy G, Riley L. Assessment and treatment of pregnant women with suspected or confirmed influenza. Obstet Gynecol 2018;132(753):169-173

2 Di Mascio D, Khalil A, Saccone G, et al. Outcome of coronavirus spectrum infections (SARS, MERS, COVID-19) during pregnancy: a systematic review and meta-analysis. Am J Obstet Gynecol MFM 2020;2(02):100107. Doi: 10.1016/j.ajogmf.2020.100107

3 Rosenberg ES, Tesoriero JM, Rosenthal EM, et al. Cumulative incidence and diagnosis of SARS-CoV-2 infection in New York. Ann Epidemiol 2020;48:23-29.e4

4 Price-Haywood EG, Burton J, Fort D, Seoane L. Hospitalization and mortality among Black patients and White patients with Covid19. N Engl J Med 2020;382(26):2534-2543

5 Azar KMJ, Shen Z, Romanelli RJ, et al. Disparities in outcomes among COVID-19 patients in a large health care system in California. Health Aff (Millwood) 2020;39(07):1253-1262

6 Sutton D, Fuchs K, D’Alton M, Goffman D. Universal screening for SARS-CoV-2 in women admitted for delivery. N Engl J Med 2020; 382(22):2163-2164

7 Vintzileos WS, Muscat J, Hoffmann E, et al. Screening all pregnant women admitted to labor and delivery for the virus responsible for coronavirus disease 2019. Am J Obstet Gynecol 2020;223(02): 284-286

8 Campbell KH, Tornatore JM, Lawrence KE, et al. Prevalence of SARS-CoV-2 among patients admitted for childbirth in southern Connecticut. JAMA 2020;323(24):2520-2522

9 Connecticut DPH. COVID-19 Cases and Deaths by Race/Ethnicity. Connecticut Open Data Published 2020. Accessed November 19, 2021 https://data.ct.gov/Health-and-Human-Services/COVID-19Cases-and-Deaths-by-Race-Ethnicity/7rne-efic/data 
10 Goldfarb IT, Clapp MA, Soffer MD, et al. Prevalence and Severity of Coronavirus Disease 2019 (COVID-19) Illness in Symptomatic Pregnant and Postpartum Women Stratified by Hispanic Ethnicity. Obstet Gynecol 2020;Publish Ah(02):2019-2021

11 Emeruwa UN, Spiegelman J, Ona S, et al. Influence of race and ethnicity on severe acute respiratory syndrome coronavirus 2 (SARS-CoV-2) infection rates and clinical outcomes in pregnancy. Obstet Gynecol 2020;136(05):1040-1043

$12 \mathrm{R}$ Foundation for Statistical Computing. R: A Language and Environment for Statistical Computing. R Foundation for Statistical Computingwww.R-project.org. Accessed November 19, 2021

13 McKinley J. New York Has Roughly 5\% of Coronavirus Cases Worldwide. 2022https://www.nytimes.com/2020/03/22/nyregion/Coronavirus-new-York-epicenter.html. Accessed November 19, 2021

14 Coronavirus on Long Island. March 2020 https://abc7ny.com/ coronavirus-long-island-march-2020-news-nassau-county-update/6100993. Accessed November 19, 2021
15 Figueroa JF, Wadhera RK, Lee D, Yeh RW, Sommers BD. Communitylevel factors associated with racial and ethnic disparities in COVID19 rates in Massachusetts: study examines community-level factors associated with racial and ethnic disparities in COVID-19 rates in Massachusetts. Health Aff (Millwood) 2020;39(11):1984-1992

16 Papon S, Robert-Bobee I. Une hausse des décès deux fois plus forte pour les personnes nées à l'étranger que pour celles nées en France en mars-avril 2020 [An increase in deaths twice as high in March-April 2020 for people born abroad compared to those born in France]. Published online July 7, 2020. https://www.insee.fr/fr/ statistiques/4627049

17 Selden TM, Berdahl TA. COVID-19 and racial/ethnic disparities in health risk, employment, and household composition. Health Aff (Millwood) 2020;39(09):1624-1632

18 Baud D, Greub G, Favre G, et al. Second-trimester miscarriage in a pregnant woman with SARS-CoV-2 infection. JAMA 2020;323 (21):2198-2200 